\title{
Modeling of mobile channels using TIMS in IT education
}

\author{
Martin Rakús, Peter Farkaš and Tomáš Páleník \\ Department of Digital and Wireless Communications, Faculty of Electrical \\ Engineering and Information Technology, Institute of Multimedia ICT, \\ Slovak University of Technology in Bratislava, Bratislava, Slovakia
}

\begin{abstract}
Purpose - The purpose of this paper is to directly link information technology (IT) education with real-world phenomena.

Design/methodology/approach - The selected objectives are achieved by modeling line of sight (LOS) and nonline of sight (NLOS) mobile channels using corresponding distributions. Within the described experiments, students verify whether modeled generators generate random variables accordingly to the selected distribution. The results of observations are directly compared with theoretical expectations. The methodology was evaluated by students via questionnaires.
\end{abstract}

Findings - The results show that the proposed methodology can help graduate or undergraduate students better comprehend lectured material from mobile communications or mathematical statistics.

Originality/value - The hands on experience using the EMONA system make the approach original.

Keywords Mobile channel modeling, Random variable, Statistical distribution, $C H I^{2}$ distribution,

Rice distribution, Rayleigh distribution

Paper type Research paper

\section{Introduction}

Over the last two decades, many countries have observed the student's decreasing interest in enrolling in technical university or college programs. This trend still continues in spite of the growing needs for technically skilled people required by increasing mobile communications and evolving information communication technology (ICT) applications. In our experience, this aversion towards technical courses often stems from aversion to mathematics. On the other hand, to study and to understand technology, e.g. communication technology, without a solid underlying mathematical background is impossible. To address this problem, a system of modeling experiments in undergraduate and graduate courses has been applied in the Institute of Multimedia ICT at the Faculty of Electrical Engineering and Information Technology in the Slovak University of Technology "M-ICT-FEI-STU" in Bratislava, Slovakia. These experiments are based on the Telecommunications Instructional Modeling System (TIMS) [1] made by the EMONA company, located in Sydney, Australia. A reasonably large set of TIMS experiments based on pedagogical experience aimed at increasing the interest of students in telecommunications was created: [2-8]. These laboratory experiments were written with the emphasis on their direct use by the teacher without requiring additional information

(C) Martin Rakús, Peter Farkaš and Tomáš Páleník. Published in Applied Computing and Informatics. Published by Emerald Publishing Limited. This article is published under the Creative Commons Attribution (CCBY 4.0) licence. Anyone may reproduce, distribute, translate and create derivative works of this article (for both commercial and non-commercial purposes), subject to full attribution to the original publication and authors. The full terms of this licence may be seen at http://creativecommons. org/licences/by/4.0/legalcode

This work was supported by the Slovak Research and Development Agency under the Contract no. APVV-19-0436, and it was also supported by the Scientific Grant Agency of the Ministry of Education of Slovak Republic and Slovak Academy of Sciences (Grant no: VEGA 1/0 477/18).
Received 19 April 2021 Revised 25 November 2021 Accepted 13 December 2021 
sources. They provide the basic theory necessary to understand the given topics. Condensed versions of the experiments are given in [9]. They mainly serve teachers as quick links to specific topics or may inspire further ideas about experiment expansions. The main advantage of TIMS is the opportunity for students to perform hands-on experiments, which can immediately connect their observations of the real system behavior with the theoretical expectations. At M-ICT-FEI-STU TIMS, experiments serve as a supplementary teaching material to accompany the following subjects: digital communications I and II and design of wireless telecommunication networks. The following paper is aimed at addressing selected topics from digital communications, namely mobile channel modeling. It can be also useful for math (or other technical) subjects dealing with statistical theory, namely with $C H I^{2}$ distribution. At the same time, it can also serve as a math support for students from other scientific areas, e.g. physics or chemistry. The paper is organized as follows. In Section 2, the motivation, target objective and description of the proposed experiment setup are given. The theoretical background of the presented experiment is given in Section 3. Details of the described experiment together with the graphical output are given in Section 4. A description of the methodology for evaluating the contribution of the presented experiments is given in Section 5. Section 6 concludes the paper.

\section{Motivation and description of the experiment setup}

It is a well-known practical experience that students will better retain the outcome of some experiment if they can carry out that experiment themselves. At the same time, this process significantly helps students to link studied theoretical background with practical real-world experimental findings. Frequently, complex maths can be explained using simple and easily understandable signals representing the lectured theory. The next important outcome of this practical approach is that when the experiment is carried out correctly, its directly measurable outcome matches the theoretical expectation and thus gives students a practical demonstration that the theory is valid also when implemented in a real world system. This way students can prove by themselves that frequently "widely" looking theories really work.

To present this concept, experiments analyzing the main statistical properties of Rice and Rayleigh distribution were chosen. Both distributions are the key distributions in mobile channel analysis. In communication theory, these distributions play an important role in wireless channel modeling. Nowadays, wireless communications are rapidly expanding due to new emerging $5 \mathrm{G}$ technologies enabling ubiquitous communications between a wide variety of different sinks and sources. Besides traditional human-to-human communication, machine-to-human or machine-to-machine communications with all its practical realizations (IoT, V2X, [...]) will expand in the near future. Therefore, modeling and simulating mobile channels in the context of rising new wireless technologies and applications is a vital topic.

In the following experiments, random variable generators with central and non-central $\mathrm{CHI}^{2}$ distributions are built. Out of those two distributions, parameters for Rice and Rayleigh distribution were derived. Based on the collected set of signal samples, the main statistical properties of generated random signals are calculated. To demonstrate that the generated signals have Rice and Rayleigh distributions, histograms of the collected data are compared against theoretical probability density functions (PDFs).

The experiments will be performed using the EMONA TIMS 301 modeling system [1]. The proposed experiments can be carried out in several different ways, depending on the possibilities of a particular institution. The ideal case is when each student can perform an experiment by themselves. This is the most financially demanding solution. Substantial 
cost saving can be achieved by creating a so called "demonstration" scenario described in [10]. In this case, there is only one set of measuring equipment operated either by the teacher or by students. The measured signals are projected on a screen. This way the other students can observe the presented signals and read measured values. The other solution is to use the Emona Telecoms-Trainer 101 (ETT-101) [1], which costs substantially less than the TIMS 301 system. For each of the described experiments, two sets of ETT-101 (still costing less than one TIMS 301 system) are necessary. The last solution is to use the TutorTIMS software (the least expensive alternative of the mentioned three). In this case, students are not working with real signals, but they simulate experiments using software which partially separates them from hands-on experience. The prerequisites for successfully performing the described experiments are an understanding of the terms describing random variables, such as mean, variance and standard deviation, and testing of statistical hypotheses. Further details can be found in [11, 12] and [13].

\section{A little bit of theory}

The following notes (limited only to the later mentioned experiments and its analysis) are taken from [11].

\subsection{Rice distribution}

In the next section, the main parameters of Rice distribution will be described. This distribution describes multi-path channels with multiple reflected paths plus a line of sight (LOS) component. This type of distribution is closely related to Gaussian distribution; actually it is its transformation. Let,

$$
Y=X_{1}^{2}+X_{2}^{2}
$$

where $X_{1}$ and $X_{2}$ are statistically independent and identically distributed Gaussian random variables with nonzero means $m_{1}, m_{2}$ and common variance $\sigma^{2}$. A new random variable

$$
Z=\sqrt{Y}=\sqrt{X_{1}^{2}+X_{2}^{2}}
$$

has Rice distribution with the PDF:

$$
p_{Z}(z)=\frac{z}{\sigma^{2}} e^{-\frac{\left(z+s^{2}\right)}{2 \sigma^{2}}} I_{0}\left(\frac{z s}{\sigma^{2}}\right), \quad z \geq 0
$$

where $s^{2}$ is the noncentrality parameter of the distribution:

$$
s^{2}=\sum_{i=1}^{2} m_{i}^{2}
$$

and $m_{i}$ is the nonzero mean of $X_{i} \cdot I_{0}(x)$ is a zero-order modified Bessel function of the first kind. The cumulative distribution function $(\mathrm{CDF})$ of the Rice distribution is as follows:

$$
F_{Z}(z)=1-Q_{1}\left(\frac{s}{\sigma}, \frac{z}{\sigma}\right) \quad z \geq 0
$$

where $Q_{1}(a, b)$ is Marcum's $Q$ function: 


$$
Q_{1}(a, b)=e^{-\left(a^{2}+b^{2}\right) / 2} \sum_{k=0}^{\infty}\left(\frac{a}{b}\right)^{k} I_{k}(a, b) \quad b>a>0
$$

$\mathrm{CDF}$, sometimes denoted as the error function, may also be expressed in terms of the complementary error function denoted as $F_{c}(z)$, then

$$
F(z)=1-F_{c}(z)
$$

The first two moments are as follows:

$$
\begin{gathered}
E(Z)=\sigma \sqrt{\frac{\pi}{2}} L_{1,2}\left(\frac{-s^{2}}{2 \sigma^{2}}\right) \\
\sigma_{z}^{2}=2 \sigma^{2}+s^{2}-\frac{\pi \sigma^{2}}{2} L_{1,2}^{2}\left(\frac{-s^{2}}{2 \sigma^{2}}\right)
\end{gathered}
$$

where $L_{1,2}(x)$ is a Laguerre polynomial:

$$
L_{1,2}(x)=e^{\frac{x}{2}}\left[(1-x) I_{0}\left(\frac{-x}{2}\right)-x I_{1}\left(\frac{-x}{2}\right)\right]
$$

\subsection{Rayleigh distribution}

Similarly as in 3.1, the main parameters of Rayleigh distribution can be listed. The PDF of Rayleigh distribution is as follows:

$$
p_{Z}(z)=\frac{z}{\sigma^{2}} e^{\frac{-2^{2}}{\sigma^{2}}}, \quad z \geq 0
$$

The first two moments are as follows:

$$
E(Z)=\sqrt{\frac{\pi}{2}} \sigma
$$

and

$$
\sigma_{z}^{2}=\left(2-\frac{1}{2} \pi\right) \sigma^{2}
$$

The CDF of Rayleigh distribution is as follows:

$$
F_{Z}(z)=1-e \frac{-z^{2}}{2 \sigma^{2}} \quad z \geq 0
$$

As in the case of Rice distribution, CDF may also be expressed in terms of the complementary error function $F_{c}(z)$ defined in $(7)$.

\section{Experiment setup}

In this section, the experimental setup enabling us to build models of random variable generators will be described. More details of the presented experiment can be found in [14, 15]. Signal samples are collected by means of a digital oscilloscope. Based on the collected sets of samples, the main signal's statistical properties will be calculated. Universal hypothesis-testing methods will be used to show that the constructed random signal generators generate signals with the analyzed distribution. This fact will also be supported by comparing the shape of a theoretical PDF against an empirically constructed histogram. As was mentioned in Section 2, the following experiments will be carried out using the TIMS 301 modeling system. 


\subsection{Modeling Rice distribution}

At first, a random signal generator generating signal with non-central $\mathrm{CHI}^{2}$ distribution is built. In order to obtain signal samples with Rice distribution, the square root of signal with non-central $\mathrm{CHI}^{2}$ distribution is performed by calculation (2). The main statistical parameters of the generated random signal: mean, variance and standard deviation will be determined from the set of calculated values. Based on the collected data set and by using universal hypothesis-testing methods, it will be shown that the generated data set has Rice distribution. A block diagram of the measurement set-up is shown in Figure 1.

4.1.1 Measuring the main statistical parameters of the generated random signal. The attenuators on both NOISE GENERATORS modules are set to +20 [dB]. The NOISE GENERATORS modules provide a random signal whose distribution is relatively close to Gaussian [16]. The output bandwidth of the TIMS noise generator is approx. 430 [kHz]. In order to use only the flat part of the noise spectrum (to approximate AWGN), the outputs of the noise generators are fed to $60 \mathrm{kHz}$ low-pass filters (LPFs). Using an oscilloscope's measurement options, it is possible to measure the average $\mathrm{DC}$ and $\mathrm{AC}$ value of the signal at the output of LPF 1 (see Figure 1) - check point 2 on the block diagram. Suitable oscilloscope settings are as follows: measurement: filtered; maximum number of samples per scope trace: 50 000; trig.: ext; time base: $100 \mathrm{~ms}$ /div; time base: $\times 1$; vertical scale: $\pm 10 \mathrm{~V} \mathrm{DC}$. Measurement setting: DC voltage; whole trace; display current value and average; no. of readings: 200; AC voltage; whole trace; display current value and average; no. of readings: 200 . This setup

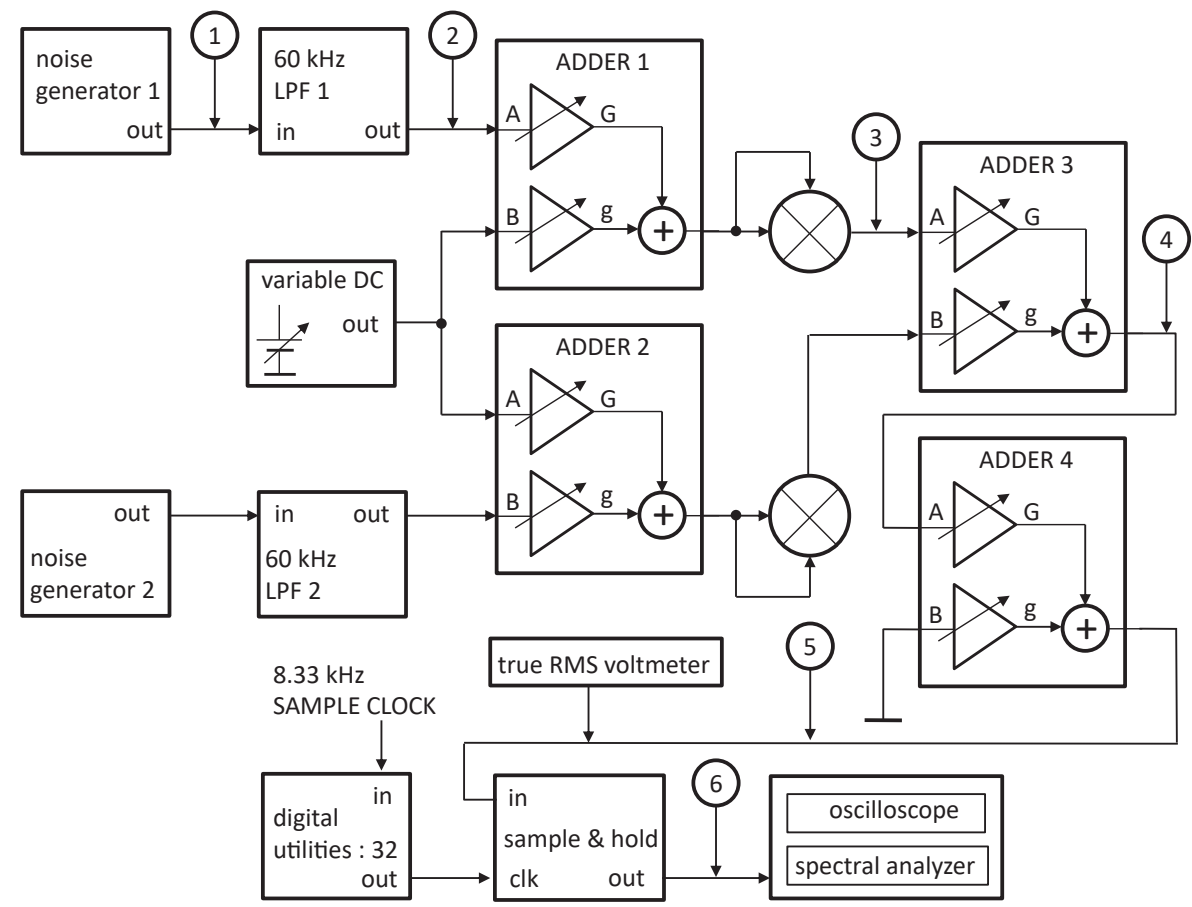

Note(s): List of necessary modules: ADDER x 4, DIGITAL UTILITIES, INTEGRATE \& DUMP, MULTIPLIER x 2, NOISE GENERATOR x 2, 60kHz LPF x 2, WIDE BAND TRUE RMS METER. A MASTER SIGNALS module (providing $8.33 \mathrm{kHz}$ SAMPLE CLOCK) and a VARIABLE DC module are built into the TIMS301 system
Modeling of mobile channels 
provides an adequate accuracy. The displayed $\mathrm{DC}$ voltage is the average voltage during one complete cycle. The displayed $\mathrm{AC}$ voltage is the root mean square $(\mathrm{rms})$ sum of the reading minus the $\mathrm{DC}$ voltage for one complete cycle. Therefore, the $r m s$ value of the random signal is equal to

$$
r m s=\sqrt{D C^{2}+A C^{2}}
$$

In order to increase the accuracy, from now on the average values of the analyzed signals will be used. All entries in (16) and (17) are average values. Using the gain control on the $60 \mathrm{kHz}$ LPF 1 module, the output $\mathrm{AC}$ voltage should be set to approx. $1\left[\mathrm{~V}_{\text {rms }}\right]$ (check point 2 in Figure 1). The output voltage of VARIABLE DC module is set to approx. -1 [V]. The gain of input $A$ on ADDER 1 is set to unity. With gain control $g$, the level of the DC component at the adder output is set to approx. $250[\mathrm{mV}]$. Let particular measured values of signal components of the signal $X$ be

$$
X_{r m s D C}=0.2779[\mathrm{~V}] \quad X_{r m s A C}=1.059[\mathrm{~V}]
$$

The measured values of the average $\mathrm{DC}$ and $\mathrm{AC}$ components of the random signal $X$ are recorded. Using the gain control on the $60 \mathrm{kHz}$ LPF 2 (see Figure 1) and gains: $G$ and $g$ on ADDER 2 it is necessary to set the values of the DC and AC components of the random signal $Y$ (at the output of ADDER 2) as close as possible to the values for the signal $X$. Let particular measured values of signal components of the signal $Y$ be

$$
Y_{r m s D C}=0.2723[\mathrm{~V}] \quad Y_{r m s A C}=1.052[\mathrm{~V}]
$$

The mean and standard deviation of the analyzed signals are as follows:

$$
\begin{aligned}
& X_{r m s D C}=m_{X}=m_{1}=0.2779[\mathrm{~V}] \\
& Y_{r m s D C}=m_{Y}=m_{2}=0.2723[\mathrm{~V}]
\end{aligned}
$$

and

$$
\begin{aligned}
X_{r m s A C} & =\sigma_{X}=1.059[\mathrm{~V}] \\
Y_{r m s A C} & =\sigma_{Y}=1.052[\mathrm{~V}]
\end{aligned}
$$

For further calculations (based on the parameters of used noise generators), from now on the following standard deviation will be used:

$$
\sigma=\frac{\sigma_{X}+\sigma_{Y}}{2}=1.0555[\mathrm{~V}]
$$

The filtered noise is squared by the MULTIPLIER module. The coupling on both multipliers should be switched to DC. The squared filtered noise is summed in the ADDER 3 module (see Figure 1). The gains of both inputs of the ADDER 3 module are set to unity. Since the adder operates in inverted mode, the signal must be inverted back. The second inversion is performed by the ADDER 4 module. The gain $G$ on ADDER 4 is set to approx. 12 o'clock and gain $g$ is set fully counter clockwise. The summed squared filtered noise is sampled by the INTEGRATE \& DUMP module. The printed circuit board mount rotary switch $S W 1$ on the INTEGRATE \& DUMP module is switched to the "S\&H1" position. To eliminate transients between successive samples, the "S\&H1" circuit is clocked with a slow clock signal. The clock signal is derived from $8.3[\mathrm{kHz}$ SAMPLE CLOCK (by division 32) using the DIGITAL UTILITIES module. Thus clock frequency of this experiment is as follows:

$$
f_{c l k}=\frac{100[\mathrm{kHz}]}{12} \times \frac{1}{32} \doteq 260.42[\mathrm{~Hz}]
$$

The values of random signal samples are collected using the oscilloscope's cursor, see Figure 2. 


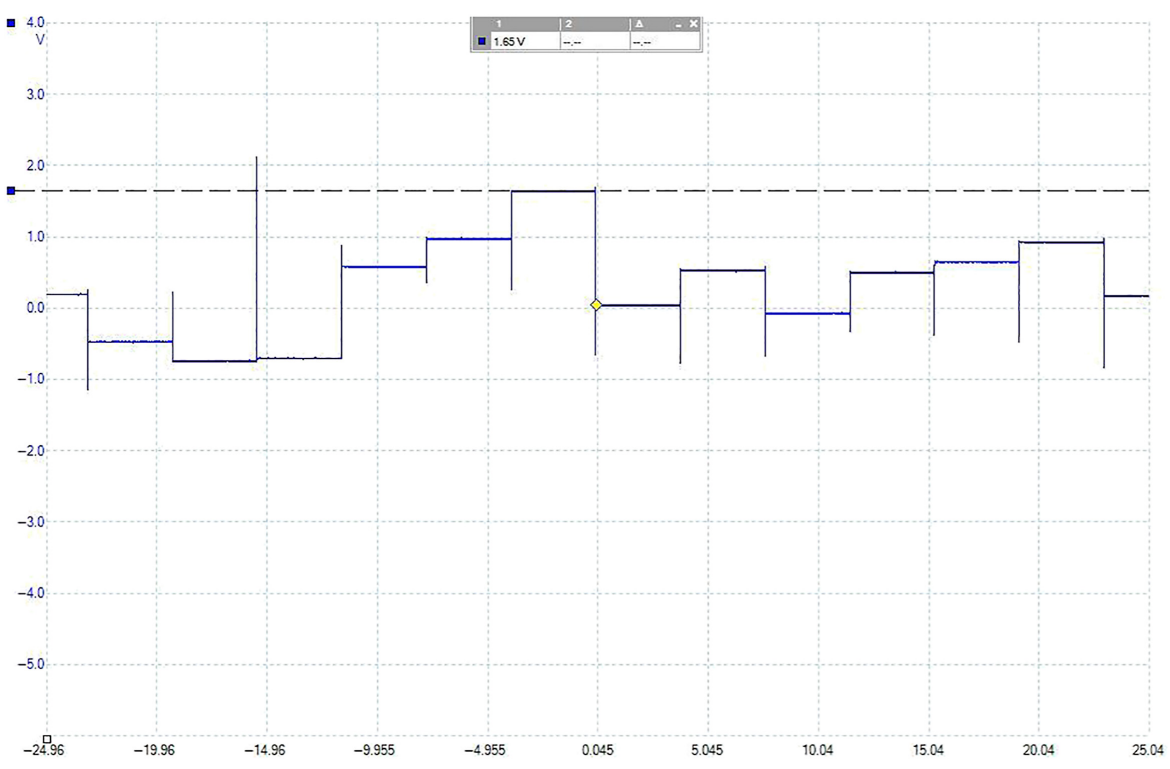

Modeling of mobile channels

Figure 2. Random signal samples-check point 6 on the block diagram

Sample values are measured using the horizontal cursor (value is displayed at the top of the screen, e.g. $x=1.65$ [V]), see Figure 2. With this setting, 13 samples fit on one screen. Using the cursor has the advantage that the effect of noise can be eliminated immediately by placing the cursor "in the middle" of the sample value. Taking 130 measurements with a cursor takes app. 15 min. A sample of collected data is in Table 1. Collected data are first square root and then corrected.

Based on the collected data, two main statistical parameters can be estimated: mean and variance. The following numerical examples are derived from the collected 130-element dataset using the above described procedure. Based on (4) and (18), the non-centrality parameter is as follows:

$$
s^{2}=\sum_{i=1}^{2} m_{i}^{2}=0.151376
$$

The estimated population mean (denoted $m_{Z}$ ) of Rice distribution based on $\sigma$ of the original noise generators using (8), (10) and (20) can be estimated:

$$
m_{Z}=\sigma \sqrt{\frac{\pi}{2}} e^{-\frac{s^{2}}{4 \sigma^{2}}}\left[\left(1-\frac{s^{2}}{2 \sigma^{2}}\right) I_{0}\left(\frac{s^{2}}{4 \sigma^{2}}\right)-\frac{-s^{2}}{2 \sigma^{2}} I_{1}\left(\frac{s^{2}}{4 \sigma^{2}}\right)\right]=1.36743
$$

\begin{tabular}{cccc}
\hline$n$ & Measured values $z_{i}$ & SQRT of measured values $z_{S Q R T}$ & Corrected values $z_{\text {icorr }}$ \\
\hline 1 & 0.006 & 0.077460 & 0.115105 \\
2 & 0.014 & 0.118322 & 0.175826 \\
3 & 0.055 & 0.234521 & 0.348498 \\
$\vdots$ & $\vdots$ & $\vdots$ & $\vdots$
\end{tabular}

Table 1 . Collected data of random signal 
The estimated sample mean (denoted $\bar{z}$ ) of a random signal based on measured data's square root can be estimated as follows:

$$
\bar{z}=\frac{1}{n} \sum_{i=1}^{n} z_{i}=0.91822
$$

where $n$ denotes number of samples (in the experiment $n=130$ ), and $z_{i}$ is the value of the $i$-th sample. By comparing (23) and (24), we see that the population mean is greater than the sample mean. This is caused by the real constants applied in the created model (e.g. multiplier has constant $\approx 0.5$ ). In order to compare the measured statistical parameters with theoretical predictions, it is necessary to compensate all realization constants by applying one single constant, denoted as $k$. Let us assume for now (it will be verified later) that the collected samples follow the Rice distribution - empirical and theoretical PDFs match (shape wise) but only means are shifted. To match means, it is necessary to multiply the square root of collected samples by $k$ to obtain corrected data, denoted as data $a_{\text {corr }}$ :

$$
\text { data }_{\text {corr }}=k \times \text { data }
$$

To find an exact value of $k$, a tray-and-error method is used to match the sample mean to the population mean. As a starting value, the reciprocal value of the multiplier's constant can be used. Let $k=1.486$, then the mean of the corrected data, denoted as $\bar{z}_{\text {corr }}=1.36447$, which matches the estimated population mean (23).

The estimated population variance (denoted $\sigma_{z}^{2}$ ) of Rice distribution based on $\sigma$ of the original noise generators using (9), (10), (20) and (22) can be estimated as follows:

$$
\sigma_{Z}^{2}=2 \sigma^{2}+s^{2}-\frac{\pi \sigma^{2}}{2}\left[\left(1-\frac{s^{2}}{2 \sigma^{2}}\right) I_{0}\left(\frac{s^{2}}{4 \sigma^{2}}\right)-\frac{-s^{2}}{2 \sigma^{2}} I_{1}\left(\frac{s^{2}}{4 \sigma^{2}}\right)\right]=0.50967
$$

Variance of the corrected data, denoted as var $_{Z \text { corr }}$ can be estimated as follows:

$$
\operatorname{var}_{\text {Zcorr }}=\frac{1}{n} \sum_{i=1}^{n}\left(\sqrt{z_{\text {icorr }}}-\bar{z}_{\text {corr }}\right)^{2}=0.46756
$$

which is relatively close to (26). From the estimated value of the population, variance standard deviation can be calculated:

$$
\sigma_{z}=\sqrt{\sigma_{z}^{2}}=0.71391
$$

and similarly, the standard deviation of the corrected sample (denotes as $\operatorname{stt}_{Z \text { corr }}$ ) is as follows:

$$
s t t d_{Z c o r r}=\sqrt{\text { var Zcorr }}=0.68378
$$

By comparing (28) and (29), it is possible to observe that the standard deviation of corrected samples matches the predicted theoretical value.

In the next section, it will be verified that the generated random signal has Rice distribution. This will be performed by testing the statistical hypothesis that the analyzed distribution has a predicted distribution of probability. The testing will be performed using a $\mathrm{CHI}^{2}$ goodness-of-fit test and a Kolmogorov-Smirnov goodness-of-fit test.

4.1.2 $\mathrm{CHI}^{2}$ goodness-of-fit test. The $C \mathrm{HI}^{2}$ goodness-of-fit test [17] is used to test if a sample of data came from a population with a specific distribution. This test is defined for the hypothesis: 
(1) $H_{0}$ - the data follow a specified distribution.

Modeling

(2) $H_{1}$ - the data do not follow a specified distribution. of mobile channels

In the next step at significance level $\alpha=0.05$, the hypothesis that the square root of the collected random signal samples from Table 1 have Rice distribution of probability (with parameters: "s" and $\sigma$ ) will be tested using the $C H I^{2}$ goodness-of-fit test.

For the $\mathrm{CHI}^{2}$ goodness-of-fit computation, the data are divided into $l$ bins and the test statistic is defined as follows:

$$
\chi_{n}^{2}=\sum_{j=1}^{l} \frac{\left(n_{j}-n \times p_{j}\right)^{2}}{n \times p_{j}}
$$

where $l$ is the number of bins, $n_{j}$ is the observed frequency for bin $j, n \times p_{j}$ is the expected frequency for $\operatorname{bin} j$ and $n$ is the sample size:

$$
n=\sum_{j=1}^{l} n_{j}
$$

The expected frequency is calculated using the following formula:

$$
n \times p_{j}=n \times\left(F\left(z_{u}\right)-F\left(z_{l}\right)\right)
$$

where $F$ is the CDF for the distribution being tested, $z_{u}$ is the upper limit for class $j$ and $z_{l}$ is the lower limit for class $j$. The expected frequency for this test should be at least 5 . The test statistic follows, approximately, a $\mathrm{CHI}^{2}$ distribution with $l-c-1$ degrees of freedom where $l$ is the number of non-empty cells, and $c$ is the number of the estimated parameters. Therefore, the hypothesis that the data are from a population with the specified distribution is rejected if

$$
\chi_{n}^{2}>\chi^{2}(1-\alpha, l-c-1)
$$

where the number: $\chi^{2}(1-\alpha, l-c-1)$ is a critical value for a test at significance level $\alpha$. Corrected sampled data are distributed into bins of width of 0.25 [V]. Note that bins on tails are combined to reach an expected frequency of at least 5 . An example of a table for calculating the $\chi_{130}^{2}$ statistic is in Table 2 .

Values of $p_{j}$ can be calculated as follows:

$$
p_{j}=F\left(z_{j u}\right)-F\left(z_{j l}\right)
$$

e.g. for bin \#3:

$$
p_{3}=F(1.0)-F(0.75) \doteq 0.1324
$$

The value of the $\chi_{130}^{2}$ statistic is the sum of the entries in the bottom row of Table 2:

$$
\chi_{130}^{2}=\sum_{j=1}^{9} \frac{\left(n_{j}-n \times p_{j}\right)^{2}}{n \times p_{j}}=10.7431
$$

Noncentral $\mathrm{CHI}^{2}$ distribution has two parameters: number of degrees of freedom and noncentrality parameter $s^{2}$. Therefore, $c=2$; thus, it is necessary to find $1-\alpha=95 \%$ quantile with $l-c-1=9-2-1=6$ degrees of freedom. To find it, tables, e.g. [18], can be used:

$$
\chi^{2}(0.95 ; 6)=12.5916
$$


$\mathrm{ACI}$

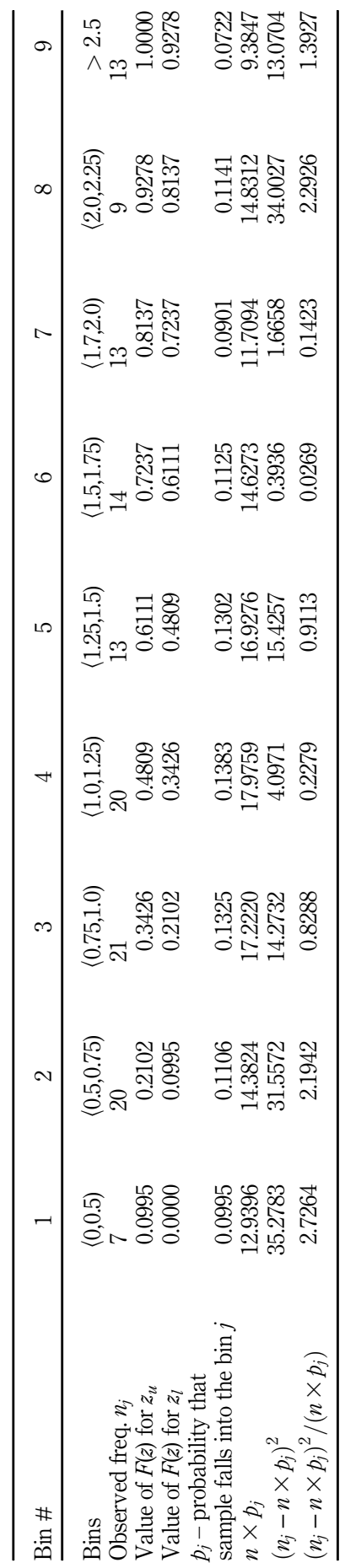

Table 2.

Intermediate data for calculating the $\chi_{130}^{2}$ statistic 
Since by applying (33),

$$
\chi_{130}^{2}<\chi^{2}(0.95 ; 6)
$$

it is possible to accept hypothesis $H_{0}$ that the analyzed random variable has Rice distribution with parameters $s=0.38907$ and $\sigma=1.0555$ at significance level $\alpha=0.05$.

4.1.3 Kolmogorov-Smirnov goodness-of-fit test. The Kolmogorov-Smirnov (K-S) test [19] is a nonparametric test for the equality of continuous, one-dimensional probability distributions that can be used to compare a sample with a reference probability distribution (one-sample K-S test) or to compare two samples (two-sample K-S test). In the next part, the hypothesis that square root of collected random signal samples from Table 1 have Rice distribution (with parameters: "s" and $\sigma$ ) will be tested at significance level $\alpha=0.05$ using the Kolmogorov-Smirnov goodness-of-fit test.

From now on let us consider a one-sample K-S test. This test is based on the empirical CDF "ECDF", denoted as $F^{*}($.$) , given that the data sample is ordered from the smallest value to the$ largest:

$$
F^{*}(z)=\frac{1}{n} \sum_{i=1}^{n} I_{z_{i} \leq z}
$$

where $I_{z_{i} \leq z}$ is the indicator function:

$$
I_{z_{i} \leq z}\left\langle\begin{array}{ll}
1 & \text { if } z_{i} \leq z \\
0 & \text { otherwise }
\end{array}\right.
$$

The K-S test is defined for the hypothesis:

(1) $H_{0}$ - the data follow a specified distribution (given by $\mathrm{CDF}$ ).

(2) $H_{1}$ - the data do not follow a specified distribution.

The K-S test statistic (a measure of difference between ECDF and CDF) is defined as follows:

$$
D_{n}=\sup _{z}\left|F^{*}(z)-F(z)\right|
$$

where $\underset{z}{\text { sup }}$ is the supremum of the set of distances. As was mentioned before, the CDF for the K-S test has to be expressly determined with all its parameters. The K-S test procedure is given as follows:

(1) order collected data from the smallest value to the largest and construct ECDF using (39),

(2) find realization $d_{n}$ of statistic $D_{n}$ :

$$
d_{n}=\max _{z}\left|F^{*}(z)-F(z)\right|
$$

(3) choose significance level $\alpha$ (probability of error that $H_{0}$ will be rejected in spite of the fact that $F(z)$ is the $\mathrm{CDF}$ of $Z$ ) and

(4) using tables, find the critical value of $D_{n, 1-\alpha}:(1-\alpha)$ quantile of $D_{n}$

(5) if

$$
d_{n}>D_{n, 1-\alpha}
$$

(at significance level $\alpha$ ) the hypothesis that data follow the specified distribution is rejected. 
A sample of the intermediate data used to calculate statistic $d_{n}$ is in Table 3.

The correction factor stays the same as was defined in Section 1). The calculated CDF based on corrected data using (5) with $s^{2}$ defined in (22), and $\sigma$ defined in (20) is denoted as $F\left(z_{i}\right)_{\text {corr }}$ Using corrected values of the data sample,

$$
d_{130}=0.05868
$$

Critical values for K-S test can be found, e.g. in [20]. For large $n>50$, the value of $D_{n}$ can be approximated:

$$
D_{130,0.95}=\frac{1.358}{\sqrt{N}}=\frac{1.358}{\sqrt{130}}=0.11910
$$

Since by applying (44),

$$
d_{130}<D_{130,0.95}
$$

it is possible to accept hypothesis $H_{0}$ that the analyzed random variable has Rice distribution with parameters $s^{2}=0.38907$ and $\sigma=1.0545$ at significance level $\alpha=0.05$. To verify whether a given data sample fits a specific distribution, various types of software (e.g. Mathematica) would be advantageous to use. Figure 3 shows a smoothed histogram of corrected data fit to Rice distribution with parameters: $s$ and $\sigma$ specified in (20) and (22).

Table 3.

Intermediate data for calculation of $d_{n}$ statistic

\begin{tabular}{ccccccc}
\hline & $\begin{array}{c}\text { Measured } \\
\text { Values } z_{i}\end{array}$ & $\begin{array}{c}\text { SQRT of Measured } \\
\text { Values } z_{i S Q R T}\end{array}$ & $\begin{array}{c}\text { Corrected } \\
\text { Values } z_{\text {icorr }}\end{array}$ & $F^{*}\left(z_{i}\right)$ & $F\left(z_{i}\right)_{\text {corr }}$ & $\left|F^{*}\left(z_{i}\right)-F\left(z_{i}\right)_{\text {corr }}\right|$ \\
\hline 1 & 0.0060 & 0.07746 & 0.11510 & 0.00000 & 0.00554 & 0.00554 \\
2 & 0.0140 & 0.11832 & 0.17583 & 0.00769 & 0.01288 & 0.00527 \\
3 & 0.0550 & 0.23452 & 0.34850 & 0.01538 & 0.04966 & 0.03427 \\
$\vdots$ & $\vdots$ & $\vdots$ & $\vdots$ & $\vdots$ \\
$\vdots$ & $\vdots$ & $\vdots$ & & & &
\end{tabular}

Figure 3.

Smoothed histogram of collected samples $(\longleftarrow)$ fit to Rice distribution (bulleted line)

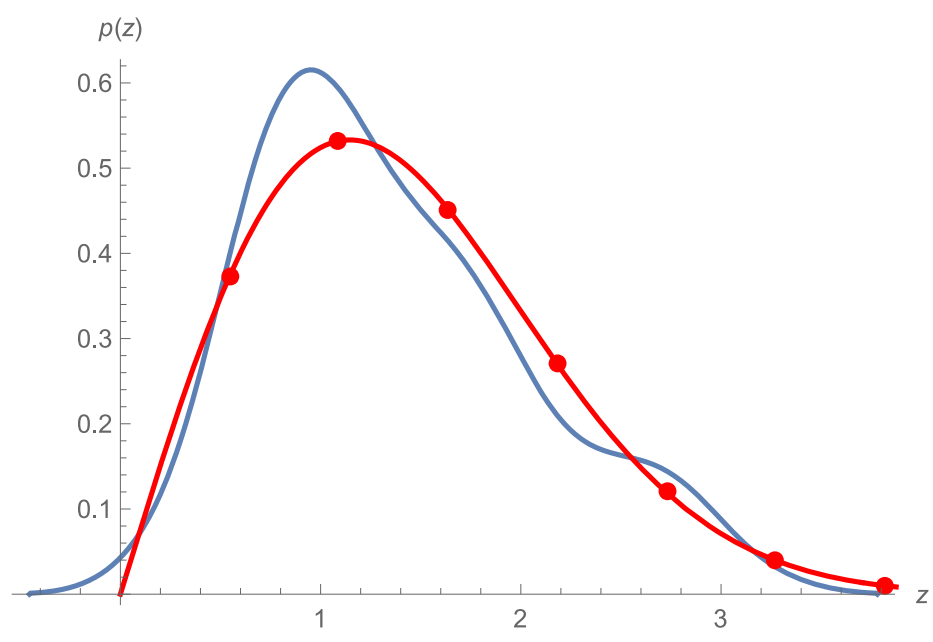




\subsection{Modeling Rayleigh distribution}

More details of the presented experiment can be found in [15]. At first, a random signal generator generating signal with central $\mathrm{CHI}^{2}$ distribution is built. In order to obtain signal samples with Rayleigh distribution, a square root of a signal with central $\mathrm{CHI}^{2}$ distribution is performed by calculation (2). A generator producing a random variable with central $\mathrm{CHI}^{2}$ distribution is very similar to the noncentral case described in section 4.1. The main difference is that particular random variables $X$ and $Y$ do have zero mean value: $m_{X}=m_{Y}=0$. A block diagram of the experiment setup is in Figure 4.

Procedures for measuring the main statistical parameters of generated random variables and goodness-of-fit tests are performed exactly the same way as in section 4.1.

\section{Methodology to evaluate the contribution of the demonstration experiments to comprehension of mobile channel modeling}

Currently, the demonstration approach as was described in [10] is an integral part of the educational process in the undergraduate and graduate courses at M-ICT-FEI-STU, namely, in subjects such as digital communications I, II and design of wireless telecommunication networks. A questionnaire was used to survey how much the demonstration experiments contributed to better understanding of the lectured theory. The questionnaire was simple, targeting two main points:

(1) Does demonstrating the experiments help students to better comprehend the lectured theory?

(2) Would students prefer to perform "hands-on" experiments (if possible)?

A total of 71 undergraduate students studying "digital communications I", 45 undergraduate students studying "digital communications II" and 31 graduate students studying "design of wireless telecommunication networks" participated in the survey. The survey was performed during the seminars. The collected responses were evaluated using a four-level Likert scale, with 4 meaning strongly agree and 1 meaning strongly disagree. The survey showed that $94.87 \%$ of participants declared that signal modeling using TIMS positively contributed to their better understanding of the lectured theory. The overall outcome of the performed survey clearly reveals that using TIMS to complement IT courses helps students to better understand the lectured matter and significantly improves IT education. The other important outcome is that students would prefer individual "hands-on" labs $(77.93 \%$ of

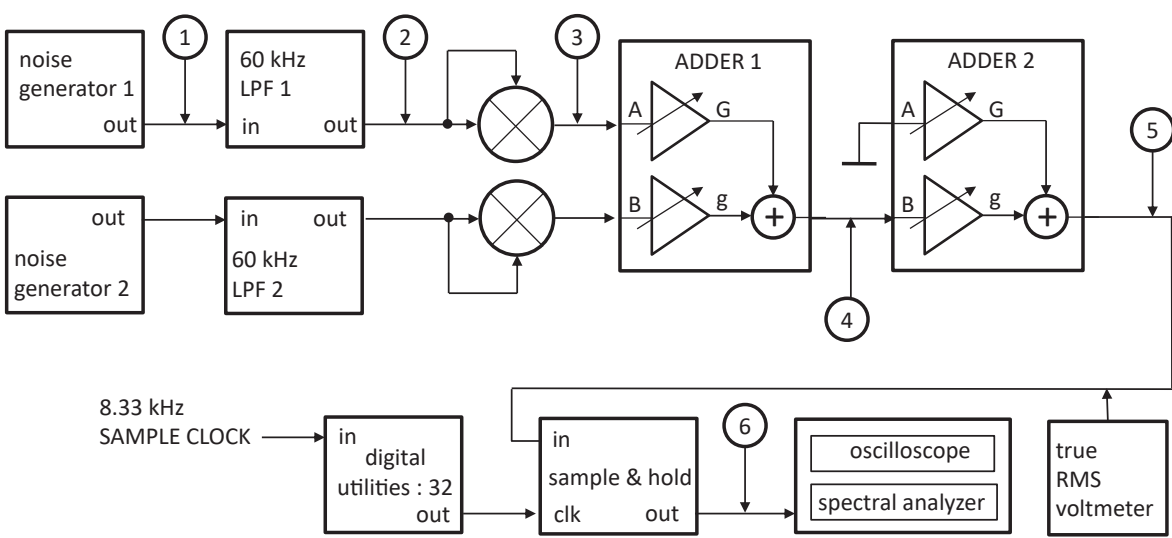

Modeling of mobile channels 
participants) and prelab learning using the simulation tool TutorTIMS $(87.6 \%$ of participants).

\section{Conclusion}

In the presented experiments, the output of a random signal generator modeling noncentral and central $\mathrm{CHI}^{2}$ distribution was sampled. In order to obtain Rice or Rayleigh distribution, the square root of collected data was calculated. Based on the calculated data, estimates of the two main statistical parameters, such as mean and variance, were performed. To test whether the generated signal had a Rice or Rayleigh distribution, two universal goodness-of-fit tests, such as $\mathrm{CHI}^{2}$ and Kolmogorov-Smirnov, were used. Both tests proved that the generated random signals followed the predicted distribution. Statistical testing of the usefulness of the demonstration experiments (as a complement to standard teaching methods) performed by the TIMS modeling system clearly supported the suitability of this approach in the education process. In the previously described experiments, students can practically "touch" the selected part of mobile channel modeling. Good accordance of measured results with the theory (see Figure 3) supports the validity of the proposed measurement methods. Our experience with this approach in the educational process helps justify modeling methods in education as an important complement to theoretical lectures.

\section{References}

1. EMONA TIMS. Homepage. [upadated: 2021]. Available at: https:/www.emona-tims.com/.

2. Rakus M., et al. Volume D4 further advanced digital experiments, communication systems modelling with TIMS. Australia: Emona Instruments Pty; 2011. 978-1-921903-00-7.

3. Rakus M. Volume D5 basic spread spectrum experiments, communication systems modelling with TIMS. Australia: Emona Instruments Pty; 2011. 978-1- 921903-01-4.

4. Rakus M. Volume D6 advanced spread spectrum experiments, communication systems modelling with TIMS. Australia: Emona Instruments Pty; 2012. 978-1-921903-09-0.

5. Rakus M. Volume D7 advanced BER experiments, communication systems modelling with TIMS. Australia: Emona Instruments Pty; 2012. 978-1-921903-10-6.

6. Rakus M, Manfredini C. Volume D8 multipath experiments, communication systems modelling with TIMS. Australia: Emona Instruments Pty; 2014. 978-1-921903-11-3.

7. Rakus M. Volume D9 RADAR signals experiments, communication systems modelling with TIMS. Australia: Emona Instruments Pty; 2015. 978-1-921903-12-0.

8. Rakus M, et al. Volume D10 OFDM experiments, communication systems modelling with TIMS. Australia: Emona Instruments Pty; 2016. 978-1-921903-13-7.

9. Hooper T, Rakus M, et al. LabSheet experiments, communication systems modelling with TIMS. Australia: Emona Instruments Pty; 2013. 978-1-921903-03-8.

10. Rakus M, Samuhelova E, Dobos J. Linking theory and practice using telecommunications instructional modelling system TIMS, educational alternatives. J Int Scientific Publications. 2014; 12: 1065-1082, ISSN 1313-2571.

11. Proakis JG. Digital communications. 4th ed. New York: McGraw-Hill; 2001. 0-07-232111-3.

12. Sklar B. Digital communications fundamentals and applications. 2nd ed. Tarzana, CA: Prentice Hall PTR; 2003.

13. Papoulis A. Probability, random variables, and stochastic processes. New York: McGraw-Hill Book Company; 1965. 
14. Rakus M. Random signal analysis: Rayleigh distribution, S \& S vol.2-03, TIMS signal \& systems advanced experiments - volume 2. Australia: Emona Instruments Pty; 2014.

15. Rakus M. Random signal analysis: Rice distribution, S \& S vol.2-04, TIMS signal \& systems advanced experiments - volume 2. Australia: Emona Instruments Pty; 2014.

Modeling of mobile channels

16. Radzyner R, Rakus M. S1-14: random signal analysis: AWGN and erfc, signal \& systems. Experiments with Emona TIMS: vol S1 - fundamentals of signals \& systems. Australia: Emona Instruments Pty; 2011. 978-1-921903-02-1.

17. Engineering statistics handbook. 1.3.5.15. Chi-square goodness-of-fit test. [updated: april 2012], Available at: www.itl.nist.gov/div898/handbook/eda/section3/eda35f.htm.

18. Engineering statistics handbook. 1.3.6.7.4. Critical values of the chi-square distribution. [updated: april 2012], Available at: https://www.itl.nist.gov/div898/handbook/eda/section3/eda3674.htm.

19. Engineering statistics handbook. 1.3.5.16. Kolmogorov-smirnov goodness-of-fit test. [updated: april 2012], Available at: www.itl.nist.gov/div898/handbook/eda/section3/eda35g.htm.

20. Real statistics using Excel. One-sample Kolmogorov-smirnov table. [updated: 2021]. Available at: https:/www.real-statistics.com/statistics-tables/kolmogorov-smirnov-table/.

\section{Corresponding author}

Martin Rakús can be contacted at: martin.rakus.stuba@gmail.com

For instructions on how to order reprints of this article, please visit our website: 